\title{
Complexities in Interpreting Perceptual Profiles among Persons with Autism Spectrum Disorders: Examples from Research on Auditory and Visual Processing
}

\author{
Armando Bertone*, Anna Bonnel, and Jacob. A. Burack
}

\section{INTRODUCTION}

The study of autism has taken an unusual path from an emphasis on psychoanalytic issues that dominated its nascent years to the current emphasis on so-called hard science. As would be expected, this vast shift in emphasis in methodology is intrinsically tied, both as an outcome and contributor, to similarly vast changes in the understanding of the disorder. With the use of applied psychophysical, experimental cognitive paradigms, and electrophysiological and brain imaging approaches to the study of information processing capabilities, the research lens shifted from the "big story" of interpersonal relationships to highly defined aspects of functioning. For example, one prevailing perspective is that atypical social and nonsocial behavior among persons with autism spectrum disorders may be the sequelae of atypical low-level information processing (1-4). This research focus inevitably leads to discussions of differences between persons with and without autism, abilities and lack of abilities, and "can" and "can't do", with an ultimate focus on detailing the perceptual characteristics of persons with ASDs. Yet, despite advances in technology, increased precision in the paradigms, and glamorization of the "hard science" of autism, the findings are often more equivocal than portrayed by the researchers. In this paper, we suggest that researchers and the consumers of their research need to provide more critical analyses and interpretations of findings from studies. In particular, we suggest that basic elements of all research in this area including task

\footnotetext{
*To whom correspondence should be addressed: Armando Bertone armando.bertone@mcgill.ca
}

complexity and participant subgrouping need to be considered in the evaluation and interpretation of the research $(5,6)$. We provide examples from research on both auditory and visual perception to highlight the extent to which study-specific factors can influence findings and limit the generalization across persons with autism.

\section{Examples from Auditory Perception}

Persons with ASD display atypical reactions to the sensory environment, including auditory hypersensitivity in response to sounds that most people find tolerable (e.g., fire truck sirens) contrasting with hyposensitivity reactions, as in the lack of response to one's own name. These "auditory paradoxes" suggest that they process sounds in an atypical fashion. Consistent with this hypothesis, auditory processing constitutes at once a remarkable strength and a relative difficulty for persons with ASD, thereby highlighting its complex, multi-level character (for a review, see $(7,8)$ ). Examples of strengths appear to be the enhanced abilities to discriminate between tones on the basis of their pitch or height value in comparison to typically developing participants matched on chronological age and IQ (9-12), whereas weaknesses include difficulties in processing words (13), and sentences (14), presented in noisy backgrounds. However, the understanding of the complex patterns of characteristics is muddied by the realization that these claims are based on findings from single studies, for which, like any study, the findings need to be interpreted carefully within the context of the task and specific subgroups of participants.

The research on the processing of speech-related stimuli among persons with ASD is an example of 
findings that appear discrepant across studies due to differences in methodology. In particular, the findings may be largely dependent on the complexity of the task and stimuli employed across studies. For example, diminished speech in noise reception thresholds (defined as the ability to correctly detect speech-stimuli presented in noise $50 \%$ of the time) were found in two studies involving age and IQ matched groups of adolescents with high-functioning autism and Asperger syndrome. In these studies high attentional demands, the participants were asked to identify words (15), and sentences (14), presented in noisy backgrounds with temporal dips (i.e., an intermittent as opposed to constant type of noise). However, with a lower-level auditory discrimination task that required participants to discriminate between pairs of vowels presented in steady pink noise, Bonnel et al. (in preparation) found intact vowel in noise discrimination abilities among high-functioning adults with autism and Asperger syndrome, matched to typically developing participants in age and IQ. The differences between these studies, both of which were focused on auditory processing of speech stimuli, highlight the importance of considering task complexity issues such as cognitive demands and the type of stimuli.

Clinical subgrouping is another crucial factor to consider in interpreting the results of studies on auditory processing. This is illustrated in the results of two recent studies on pitch processing among persons with ASD with different clinical subgroupings. For example, Heaton et al. (2008) compared the pitch discrimination abilities of a group of participants with ASD- some of whom had a history of delayed speech onset and/or mild intellectual delays- with that of a mixed group of age- and IQ-matched participant with typical development and with mild learning disabilities (16). Although the average pitch discrimination abilities of the participants with ASD did not differ from those of the participants with typical development and mild learning disabilities at the group level, enhanced pitch discrimination abilities were found for a portion of the ASD group that stood out from the rest of the group with respect to history of delayed speech onset. Using an alternative subgrouping strategy of assigning participants with high-functioning autism and those with Asperger syndrome to different experimental subgroups on the basis of the presence or absence of a history of delayed speech onset, Bonnel et al. (in preparation) found that at the group level, the participants with high-functioning autism displayed enhanced average pitch discrimination abilities, an enhancement which was not seen among the participants with Asperger syndrome.

\section{Examples from Visual Processing}

The most common behavioral method of evaluating visual perceptual functioning in different atypical populations is by measuring sensitivity to a variety of adapted global motion (integration of local motion across time and space) and global form (integration of local oriented information across space) information, argued to reflect the functional integrity of dorsal and ventral visual stream mechanisms, respectively $(17,18)$. This reasoning is motivated by findings of specialized processing of global motion and global form mechanisms believed to selectively reflect extra-striate functioning in either visual stream by specialized visual areas (e.g., global motion: MT, dorsal stream versus global form: V4, ventral stream) $(18,19)$. Based in part on studies that employ such stimuli, Braddick and colleagues (see (18) for review) advanced an influential model, referred to as the dorsal-stream vulnerability hypothesis, according to which the dorsal visual stream is more vulnerable to genetic and environmental factors, which in turn selectively affects the dorsal stream development relative to its ventral counterpart.

In ASD, dorsal-stream vulnerability has been demonstrated in part on characteristic performances referred to as perceptual signatures (20) - that are defined by a selective decrease sensitivity to global motion alongside preserved sensitivity to global form information [See Figure 1A: (21)]. Consistent with the dorsal-stream vulnerability hypothesis, we refer to these results as being "stream-specific" $(3,20)$ since results are interpreted as a neural dysfunction selectively affecting dorsal stream-related or dynamic information processing.

Although findings of decreased global motion and unaffected global form perception in ASD is consistent with a stream-related interpretation, an alternative explanation based on the complexity of the dynamic stimuli used in these experiments should not be ruled out (21). Specifically, given that the global motion stimuli used to assess dorsal stream functioning are considered to be a visually complex type of dynamic pattern, decreased sensitivity to global motion in ASD may be the result of a neuro-integrative dysfunction at a perceptual level, not necessarily specific to the dorsal stream. This argument is referred to as the complexityspecific interpretation by Bertone and Faubert (2006), who argue that in to dissociate between stream-specific (consistent with the dorsal-stream vulnerability hypothesis) and complexity-specific (consistent with neuro-integration dysfunction) interpretations, static and dynamic information processing must be simultaneously assessed in ASD using stimuli necessitating different levels of neuro-integrative 
A

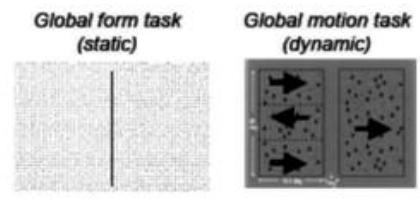

B

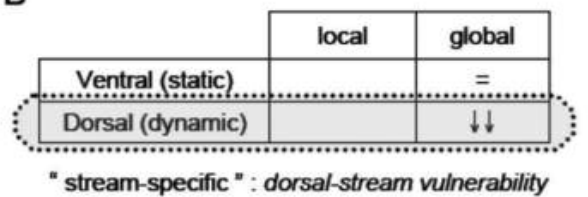

\begin{tabular}{|c|c|c|}
\hline & local & global \\
\hline Ventral (static) & & $=$ \\
\hline Dorsal (dynamic) & & $\downarrow \downarrow$ \\
\hline
\end{tabular}

ASD
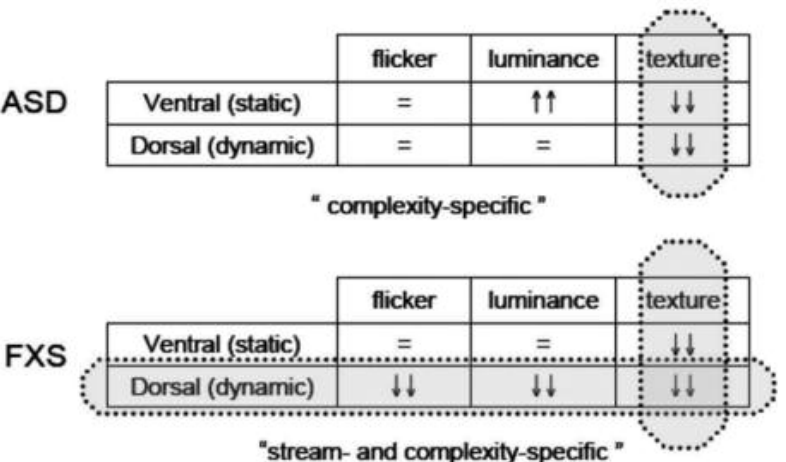

Figure 1: Schematic representation of the Spencer et al. (2000) global motion/global form paradigm (21), and the Bertone et al., (2005) static vs dynamic / luminance- vs texture-defined experimental paradigm (A) (3). Perceptual signatures for both ASD ad FXS are shown in B. When using the Spencer et al. paradigm, the perceptual signatures for ASD and FXS are the same, and therefore, a condition-specific hypothesis regarding neural alteration is not possible; both signatures are consistent with a stream-specific dysfunction, or dorsal-stream vulnerability. As represented by the downward pointing arrows $(\downarrow \downarrow)$, sensitivity to dynamic information is reduced in both ASD and FXS, with unaffected global form processing $(=)$. However, when static (orientation-identification task) and dynamic (direction-identification task) information processing is assessed in ASD and FXS as a function of stimulus complexity, two distinct perceptual signature are evidenced, with both consistent with the complexity-specific interpretation, but a selective stream-specific dysfunction for FXS (B).

processing (20). To do so, an alternate experimental paradigm is used whereby sensitivity to static and dynamic information is measured (using direction- and orientation-identification thresholds, respectively) for both luminance-defined (simple information processed in the primary visual area) and texture-defined stimuli (more complex information processed in both striate (V1) and extra-striate areas (V2/V3)) (i.e., (22-26)). Using such a paradigm, visual information processing can be defined in terms of either static/dynamic (stream-specific) or simple/complex (complexityspecific) variables in ASD. Based on Bertone et al.'s findings, the perceptual signature manifested using such a paradigm is congruent with a complexity-specific interpretation since participants with ASD were found to be less sensitive to complex, texture-defined information, whether presented in static or dynamic forms [See Figure 1B] (3). Thus, the choice of proper experimental paradigm to assess visual functioning in ASD has enabled researchers to better define the neural underpinnings of altered perception in autism, which in this case, resulted in a systematic dissociation between stream- and complexity-specific interpretations. Subsequent findings suggesting intact magnocellularrelated functioning - sensitivity to flicker information related with dorsal-stream functioning in ASD - have failed to support stream-specific visual dysfunction in $\operatorname{ASD}(27,28)$.
In addition to dissociating between hypotheses within a developmental disorder such as ASD, the choosing the proper paradigms may result in perceptual performances - or signatures - that can be used to dissociate ASD form other developmental conditions. One such model condition is fragile-x syndrome (FXS), a single-gene condition that is considered a syndromic form of ASD since between 33-67\% of children with fragile $\mathrm{X}$ will fulfill the diagnostic criteria for autism (29). As shown in Figure 1, the perceptual signatures of ASD and FXS are dissociated only when complexity variable is assessed, and therefore, only when using this paradigm can perceptual signatures be used to argue either a condition-specific neural etiology based on neural alterations underlying perceptual processing in ASD and FXS $(30,31)$. Finally, we argue that using our paradigm is advantageous over previous ones (i.e., global motion / global form paradigm) given that it allows us to define and dissociate visual information processing in terms of either static/dynamic (streamspecific) or simple/complex (complexity-specific) variables independently as a function of development. This is an important feature given that maturation of mechanisms mediating static and dynamic perception has been recently demonstrated to be differentially affected by stimulus complexity in typically developing children (32). Presented within the context of complexity- vs stream-specific interpretations, these 
results demonstrate that dorsal-stream vulnerability may only be manifested at certain ages (prior to 6 years old) under certain experimental conditions during typical development. Specifically, if a dorsal-stream vulnerability does indeed characterize typical visual developmental, it's consequences on visual performance are only manifested when (1) complex visual stimuli (i.e., texture-defined) are used to assess dorsal-stream associated functioning, (2) visual performance is assessed before about the age of six years, and (3) ventral- and dorsal-stream associated functioning is assessed using experimental paradigms at different levels of complexity.

\section{CONCLUSION}

In conclusion, the choice of experimental paradigm for assessing either auditory or visual capabilities in ASD is not only crucial for understanding the neural processes underlying atypical perception in ASD, but also for measuring performance that is specific to ASD. The latter outcome represents a potentially useful approach for dissociating ASD form other neurodevelopmental conditions at perceptual and neural levels across development.

\section{ACKNOWLEDGEMENTS}

Work on this article was supported by an operating grant for the Social Sciences and Humanities Research Council of Canada to Jacob A. Burack, and a Canadian Institutes of Health Research - Clinical Research Initiative fellowship to Armando Bertone.

\section{REFERENCES}

1. Mottron L, Burack J. Enhanced perceptual functioning in the development of autism. In: J. A. Burack TC, N. Yirmiya, and P. R. Zelazo ed. The development of autism: Perspectives from theory and research: Mahwah, NJ: Lawrence Erlbaum Associates. 2001:131-48.

2. Mottron L, Dawson M, Soulieres I, Hubert B, Burack J. Enhanced perceptual functioning in autism: an update, and eight principles of autistic perception. Journal of autism and developmental disorders. 2006 Jan;36(1):27-43.

3. Bertone A, Mottron L, Jelenic P, Faubert J. Enhanced and diminished visuo-spatial information processing in autism depends on stimulus complexity. Brain. 2005 Oct;128(Pt 10):2430-41.

4. Belmonte MK, Cook EH, Jr., Anderson GM, Rubenstein JL, Greenough WT, Beckel-Mitchener A, et al. Autism as a disorder of neural information processing: directions for research and targets for therapy. Molecular psychiatry. 2004 Jul;9(7):646-63.

5. Burack JA, Iarocci G, Bowler D, Mottron L. Benefits and pitfalls in the merging of disciplines: the example of developmental psychopathology and the study of persons with autism. Development and psychopathology. 2002 Spring;14(2):225-37.

6. Burack JA, Iarocci G, Flanagan TD, Bowler DM. On mosaics and melting pots: conceptual considerations of comparison and matching strategies. Journal of autism and developmental disorders. 2004 Feb;34(1):65-73.

7. Kellerman GR, Fan J, Gorman JM. Auditory abnormalities in autism: toward functional distinctions among findings. CNS spectrums. 2005 Sep;10(9):748-56.

8. Samson F, Mottron L, Jemel B, Belin P, Ciocca V. Can spectrotemporal complexity explain the autistic pattern of performance on auditory tasks? Journal of autism and developmental disorders. 2006 Jan;36(1):65-76.

9. Bonnel ea. 2009, in preparation.

10. Bonnel A, Mottron L, Peretz I, Trudel M, Gallun E, Bonnel AM. Enhanced pitch sensitivity in individuals with autism: a signal detection analysis. Journal of cognitive neuroscience. 2003 Feb 15;15(2):226-35.

11. Heaton P, Hermelin B, Pring L. Autism and pitch processing: a precursor for savant musical ability? . Music Perception. 1998;15:291-305.

12. O'Riordan M, Passetti F. Discrimination in autism within different sensory modalities. Journal of autism and developmental disorders. $2006 \mathrm{Jul}$;36(5):665-75.

13. Groen WB, van Orsouw L, Huurne N, Swinkels S, van der Gaag RJ, Buitelaar JK, et al. Intact spectral but abnormal temporal processing of auditory stimuli in autism. Journal of autism and developmental disorders. 2009 May;39(5):742-50.

14. Alcantara JI, Weisblatt EJ, Moore BC, Bolton PF. Speech-innoise perception in high-functioning individuals with autism or Asperger's syndrome. Journal of child psychology and psychiatry, and allied disciplines. 2004 Sep;45(6):1107-14.

15. Groen WB, van Orsouw L, Zwiers M, Swinkels S, van der Gaag RJ, Buitelaar JK. Gender in voice perception in autism. Journal of autism and developmental disorders. 2008 Nov;38(10):1819-26.

16. Heaton P, Williams K, Cummins O, Happe F. Autism and pitch processing splinter skills: a group and subgroup analysis. Autism. 2008 Mar;12(2):203-19.

17. Atkinson J, King J, Braddick O, Nokes L, Anker S, Braddick F. A specific deficit of dorsal stream function in Williams' syndrome. Neuroreport. 1997 May 27;8(8):1919-22.

18. Braddick O, Atkinson J, Wattam-Bell J. Normal and anomalous development of visual motion processing: motion coherence and 'dorsal-stream vulnerability'. Neuropsychologia. 2003;41(13):1769-84.

19. Braddick OJ, O'Brien JM, Wattam-Bell J, Atkinson J, Turner R. Form and motion coherence activate independent, but not dorsal/ventral segregated, networks in the human brain. Curr Biol. 2000 Jun 15;10(12):731-4.

20. Bertone A, Faubert J. Demonstrations of decreased sensitivity to complex motion information not enough to propose an autism-specific neural etiology. Journal of autism and developmental disorders. 2006 Jan;36(1):55-64.

21. Spencer J, O'Brien J, Riggs K, Braddick O, Atkinson J, Wattam-Bell J. Motion processing in autism: evidence for a dorsal stream deficiency. Neuroreport. 2000 Aug 21;11(12):2765-7.

22. Sperling G, Chubb C, Solomon JA, Lu ZL. Full-wave and halfwave processes in second-order motion and texture. Ciba Foundation Symposia. 1994;184:287-303.

23. Cavanagh P, Mather G. Motion: the long and short of it. Spatial vision. 1989;4(2-3):103-29.

24. Chubb C, Sperling G. Drift-balanced random stimuli: a general basis for studying non-Fourier motion perception. Journal of the Optical Society of America. 1988 Nov;5(11):1986-2007.

25. Larsson J, Landy MS, Heeger DJ. Orientation-selective adaptation to first- and second-order patterns in human visual cortex. Journal of neurophysiology. 2006 Feb;95(2):862-81.

26. Ashida H, Lingnau A, Wall MB, Smith AT. FMRI adaptation 
reveals separate mechanisms for first-order and second-order motion. Journal of neurophysiology. 2007 Feb;97(2):1319-25.

27. Pellicano E, Gibson L, Maybery M, Durkin K, Badcock DR. Abnormal global processing along the dorsal visual pathway in autism: a possible mechanism for weak visuospatial coherence? Neuropsychologia. 2005;43(7):1044-53.

28. Pellicano E, Gibson LY. Investigating the functional integrity of the dorsal visual pathway in autism and dyslexia. Neuropsychologia. 2008 Aug;46(10):2593-6.

29. Clifford S, Dissanayake C, Bui QM, Huggins R, Taylor AK, Loesch DZ. Autism spectrum phenotype in males and females with fragile $\mathrm{X}$ full mutation and premutation. Journal of autism and developmental disorders. 2007 Apr;37(4):738-47.
30. Kogan CS, Bertone A, Cornish K, Boutet I, Der Kaloustian $\mathrm{VM}$, Andermann E, et al. Integrative cortical dysfunction and pervasive motion perception deficit in fragile $\mathrm{X}$ syndrome. Neurology. 2004 Nov 9;63(9):1634-9.

31. Kogan CS, Boutet I, Cornish K, Zangenehpour S, Mullen KT, Holden JJ, et al. Differential impact of the FMR1 gene on visual processing in fragile $\mathrm{X}$ syndrome. Brain. 2004 Mar;127(Pt 3):591-601.

32. Bertone A, Hanck J, Cornish KM, Faubert J. Development of static and dynamic perception for luminance-defined and texture-defined information. Neuroreport. 2008 Jan $22 ; 19(2): 225-8$.

Anna Bonnel is a Ph.D. student in the Department of Educational and Counselling Psychology at McGill University, specializing in School and Applied Child Psychology, and is a member of the McGill Youth Study (MYST) Team. Her research is focused on auditory processing among persons with autism spectrum disorders and is supervised by Dr. Jake Burack and Dr. Laurent Mottron at Hopital Rivière-des-Prairies. Specifically, Anna is interested in how persons with autism process simple and complex sounds in comparison to typically developing individuals. Anna is also interested in the rare musical ability known as absolute pitch among persons with autism. Her research has been funded by the Fonds Québécois de la recherche sur la nature et les technologies, a training grant from the Canadian Institutes of Health Research, McGill Majors fellowships, and the Centre de Recherche Fernand Seguin. Anna is a graduate of the Autism Research training (ART) program. She is currently preparing her thesis defense.

Jacob A. (Jake) Burack is Professor of Human Development and School Applied Child Psychology in the Department of Educational and Counselling Psychology at McGill University, founder and Director of the McGill Youth Study Team (MYST), and a researcher at Hôpital Rivière-des-Prairies. His students and he work within the MYST motto of "a commitment to excellence in the study and education of all children". Their theoretical framework is that of developmental psychopathology, the interface of typical and atypical development and developmental histories. Their primary focus of study is the development of attention and cognition among typically developing children, persons with autism, and persons with specific syndromes associated with intellectual disabilities. In a second area of research, they study the academic, social, and emotional well-being of First Nations adolescents who live in northern communities.

Armando Bertone is a cognitive neuroscientist and clinical neuropsychologist completing a Canadian Institutes for Health Research - Clinical Research Initiative fellowship in the Department of Neurology and Neurosurgery at McGill University. Dr Bertone's principal research interests lie in the assessment of perceptual and sensorio-motor systems in neurodevelopmental conditions. His principal publications deal with the characterization of neural circuits underlying atypical information processing in autism and fragile $\mathrm{X}$ syndrome. Based on condition-specific perceptual performances (or signatures), he is presently developing a causal model that links genetic alteration with biologicallyplausible neural endophenotypes consistent with information processing capabilities in both conditions. He will soon be continuing his research at the Fernand-Seguin Research Center, directing the Perceptual Neuroscience Laboratory at l'Hôpital Rivière-des-Prairies. He is also a member of the CETEDUM (The University of Montreal Center of Excellence for Pervasive Developmental Disorders). 